Meta

Journal des traducteurs

Translators' Journal

\title{
Compétence rédactionnelle et compétence technique. À propos de subrogation
}

\section{Pierre Lerat}

Volume 40, numéro 2, juin 1995

Usages sociaux des termes : théories et terrains

URI : https://id.erudit.org/iderudit/003476ar

DOI : https://doi.org/10.7202/003476ar

Aller au sommaire du numéro

Éditeur(s)

Les Presses de l'Université de Montréal

ISSN

0026-0452 (imprimé)

1492-1421 (numérique)

Découvrir la revue

Citer cet article

Lerat, P. (1995). Compétence rédactionnelle et compétence technique. À propos de subrogation. Meta, 40(2), 244-249. https://doi.org/10.7202/003476ar
Résumé de l'article

Les écrits administratifs, juridiques et commerciaux ordinaires posent des problèmes de connaissances linguistiques et techniques à la fois. Les instruments existants privilégient soit les contenus soit la norme linguistique, rarement les « jeux de langage » en tant que tels. L'exemple du droit de tous les jours incite à expliciter en priorité une expertise minimale terminologique (conceptuelle), rédactionnelle (linguistique) et traductionnelle (au niveau de l'énoncé). Le cas de la subrogation illustre le besoin, les difficultés et les exigences du travail à réaliser. 


\title{
COMPÉTENCE RÉDACTIONNELLE ET COMPÉTENCE TECHNIQUE. À PROPOS DE SUBROGATION
}

PII:RRE: Li:RAT

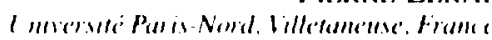

\begin{abstract}
Résumé

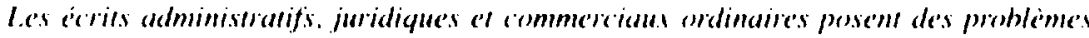
de commatssame's linguistiques al terhmiques a la fois. Le's instruments evistamts privilegient

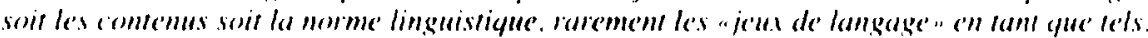

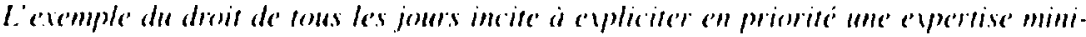

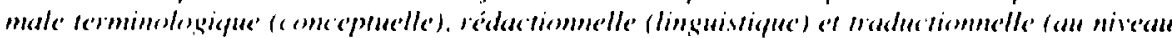

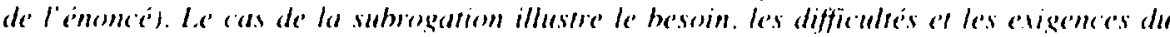

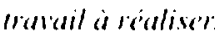

\section{INTRODUCTION}

Le problème de la circulation sociale du sens des termes est complexe. comme tout ce qui touche à la communication dans les sociétés développées. Or il y va du décodage et de l'encodage de l'expression des droits du citoyen, du locataire, de l'assuré, du contribuable, de l'assujetti, du ressortissant, du consommateur même. Aussi bien, les services publics développent une fonction d'accueil visant en partic à expliquer, et des quotidiens et revues non spécialisées ouvrent leurs colonnes à des informations qui se veulent pratiques (cas concrets, définitions, ane dites pas». etc.), mais ces bribes de savoir, qui ont leur utilité. ne valent pas un instrument de contrôle disponible au moment où l'on a à lire, à remplir ou à rédiger un document à portée administrative. juridique ou commerciale.

Cet instrument existe dans une certaine mesure, puisqu il y a des banques de données terminologiques, mais elles ne répondent pas à tous les besoins créés par des problèmes à la fois culturels et linguistiques, le public qu elles visent étant moins le citoyen que des catégories ciblées de personnel d'administrations ou d'entreprises. Quels sont les types de besoins? Comment y faire face? L'étude d'un exemple ne saurait permettre d'apporter des réponses péremptoires, mais elle peut donner une idée de l'intérêt de la tâche. el aussi de ses difficultés.

\section{POURQUIOI SUBROGATION:}

Le cas concret retenu, celui de subrogation. m'a été soumis fortuitement par une personne qui avait à remplir un imprimé d'assurance sociale. Comment expliquer le mécanisme et sa nécessité pour l'intéressé et pour son entreprise? Il ne s'agit pas de dire simplement. comme dans la vulgarisation, mais de dire adéquatement. c'est-a-dire en termes d’enjeux: au reste. on aurait du mal à mieux faire, en matière de définition, qu'un expert humaniste comme Gérard Cornu: «Substitution d une personne à une autre dans un rapport de droit en vue de permettre à la première d'exercer tout ou partie des droits qui appartiennent à la seconde" (Vorabulaire juridique 1992). Il se trouvait que je n'étais pas entièrement pris au dépourvu, venant de corédiger avec un juriste une fiche terminologique sur cette notion. mais il était en l'occurrence inapproprié de s'en tenir à la science 
juridique (la subrogation en général), à plus forte raison à une explication étymologique! Double opacité, conceptuelle et lexicale. La première peut être réduite par le recours au générique substitusion. mais la seconde? Jai montré ailleurs (Lerat 1994) que la vraie difficulté n'est pas dans le mot (que l'on ne remplacerait pas sans inconvénients par une francisation recourant à remplacememt, substitution ou toute autre approximation grossière, à plus forte raison par un néologisme d'officine) mais dans la chose: si suhdivision et interrogation ne posent pas de problèmes de communication et si subrogation en pose. c'est affaire d'expérience de vie. et non pas de motivation lexicale.

Affaire d'expérience des mots, également. C"est ce que prend en compte le fait de signaler les enchaînements syntagmatiques typiques, selon une leçon des grammaires formelles que la terminologie commence à retenir: subrogation persomnelle/rielle. subrogation comentiommelle/légale, si l'on a en vue la portée de la notion, aux fins de l'autoapprentissage. Usage effectif dans tel milieu juridique pour une même langue, si l'on veut mettre le traducteur en mesure de s'adapter à plusieurs clientèles: ici subrogation se dit Abrefung (dans le code civil allemand. le $B(; B)$, ailleurs Übergang (dans la version allemande du code suisse des obligations, l'Obligationenrecht), ailleurs encore Rechtnathfolge (à la Curia, Cour de Justice de la Communauté européenne).

Perplexité de l'assuré social, envie d'en savoir plus, besoin de reformuler dans sa langue, exigence d'une équivalence (conditionnelle) en langue étrangère: peut-on d'emblée viser multifonctionnel pour répondre à des préoccupations aussi diverses, ou bien est-on condamné à une prolifération exponentielle de bases de données terminologiques soumises aux exigences forcément particulières de tel donneur d'ordre?' Grave question. qui est plus préoccupante encore, peut-être, que les difficultés techniques (de mise en compatibilité) et juridiques (de copyright). Mais pour l'heure, voyons comment expliquer la subrogation dans un document encore plus banal que la feuille d'assurance maladie: le contrat d'assurance automobile.

\section{LA SUBROGATION DF, L;ASSIIREUR}

Un exemple de grande portée pratique. en matière de subrogation. est celui où l'assureur obtient la possibilité de défendre les droits de la victime contre des liers. Ce transfert limité de droits lui est reconnu de fait par la simple signature de la police d'assurances, qui est censée présupposer la lecture et l'approbation de cé document. La disposition du cole français des assurances renduc applicable par cette signature est la suivante: "l'assureur qui a payé l'indemnité d'assurance est subrogé. jusqu à concurrence de cette indemnité. dans les droits et actions de l'assuré contre les tiers qui, par leur fait, ont causé le dommage ayant donné lieu à la responsabilité de l'assureur": en français de simple assuré. cela permet à l'assureur de se faire rembourser en use relournant" (c'est plus ou moins le sens judiciaire d'action) contre "ladversaire" après vous avoir indemnisé. Comment l'assureur peut-il expliquer la chose"? Oralement, d'abord, par exemple de la façon approximative mais familière dont je viens de le faire. Par écrit, auquel cas deux possibilités sont en concurrence:

- une note explicative (qui a encore moins de chances d'être lue que les «petites lettres" du contrat lui-même): ainsi. la personne qui avait à remplir une uattestation de salaire pour le paiement des indemnités journalières maladie el maternité" de la sécurité sociale et qui était embarrassée par la mention ademande de subrogation en cas de maintien de salaire" sur l'imprimé n" 60-378.3 n'avait probablement pas fait l'effort de consulter. au verso. une "notice d'utilisation" yui dit très bien les choses avec les mots de tous les jours: "En cas de maintien total ou partiel du salaire, lemployeur peut demander yue les indemnités journalières dues à l'assuré lui soient versées directement. dans la mesure où 
le salaire maintenu est d'un montant au moins égal aux dites indemnités pour la période considérée. Dans ce cas l'assuré doit autoriser l'employeur à percevoir ses indemnités."

- une formulation aussi concrète que possible de la clause légale (solution la plus réaliste): par exemple. un effort louable de pédagogie est fait par la Mutuelle assurance des instituteurs de France (MAIF), qui reformule de la façon suivante la disposition codifiée: "Conformément à l'article L 121-12 du Code des Assurances, la Sorciétí qui a payé l'indemnité d'assurance est subrogée jusqu'à concurrence de cette indemnité dans les droits et actions de l'assuré contre les tiers qui, par leur fait. ont causé le dommage ayant donné lieu au paie'me'nt."

Comme on le voit, les changements portent seulement sur des termes utilisés au début et à la tin: "l'assureur" devient “la Société» el "la responsabilité de l'assureur" devient "le paiement". La première substitution résulte d'une anaphore à laquelle le lecteur est habitué quand sa lecture en est arrivée à l'article 1.3 intitulé Subrogation: Ia Société est une abréviation du nom du statut juridique de la MAIF, "société d'assurance à forme mutuelle à cotisations variablesm. La seconde est plus intéressante, car elle est purement juridique: parmi tous les aspects de la responsabilité de l'assureur, ce qui est pertinent pour l'assuré, en l'occurrence, est uniquement la forme très particulière d'implication de celle-ci que constitue le paiement de l'indemnité prévue par le contrat (puisqu' on ne s'assure que pour cela).

\section{COMMENT TRAITER SUBROGATION DANS UN OUTII. DAIDE. A LA REIDACTION EET A LA TRADUCTION?}

Une première leçon de l'exemple étudié est que suhrogatrion n'est qu'une clé documentaire dans la police d assurance: l'article 13 utilise la proposition grammaticale "l'assureur est subrogé dans les droits et actions de l'assuré". ce qui est plus explicite dans la mesure où l'on voil qui donne la subrogation et qui en est le bénéficiaire. Encore faut-il que le sens d'action (en justice) soit compris, mais observons au passage que lexpression verbale subroger est plus propre que le substantif à expliciter les actants et les enjeux: de fait, il y a lieu de sortir d'une tradition de la lexicographic à orientation terminologique qui se caractérise par la prise en compte privilégiée des noms. ce qui contribue à entretenir la confusion entre "nom" (noum) et "dénomination" (name'). Lidéal semble être de répartir l'information sur plusieurs entrées conçues comme complémentaires, puisque le substantif est indispensable comme clé documentaire et le verbe comme mise en relation des arguments (voir Roberts 1990):79).

Dans le même esprit. on peut rompre avec une autre tradition. celle d'un découpage en domaines quelque peu apriorique ou systématique. dés lors que l'usager $n^{\circ}$ a pas à passer par le filte d'un thesaurus de documentation: pourquoi ne pas concevoir une approche par types de curiosités? Dans ce cass, la subrogation pourra être abordée de façon différente selon qu il sagit d'assurances (visée pratique) ou de droit (visée doctrinale). Une gestion de type homonymique ne pose aucun problème, même en cas de redondance partielle de l'information, dans une base de données relationnelle: il suffit de faire choix d'une clé composite a trois ou quatre champs. car il n'y a pas d'exemples d'emplois terminologiques d'une même expression qui ne se distinguent soit par le domaine. soit par les dérivés pertinents (la série terminologique au sens de Chabridon et Lerat 199.3), soit par la catégorie grammaticale. soit par la distribution syntaxique, à tout le moins.

Dans un ouvrage comme le Dictiommaire juridique, où lat concision est de rigueur. l'effort pour être multifonctionnel conduit au tout-en-un. Sur base de données, il y a 
probablement avantage au contraire à séparer non pas des niveaux abstraits de compétence mais des réponses à des attentes distinctes. À titre d'exemplè, voici comment une aide à la rédaction et à la traduction d'actes juridiques, administratifs et commerciaux conçue à Paris XIII répartit sur trois enregistrements la matière de l'article du dictionnaire:

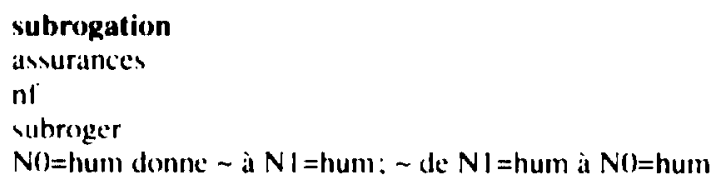

Par rapport à ces informations, une liche plus "scientifique» (plus universitaire, plus doctrinale. de portée générale) pourra avoir un contenu du type suivant:

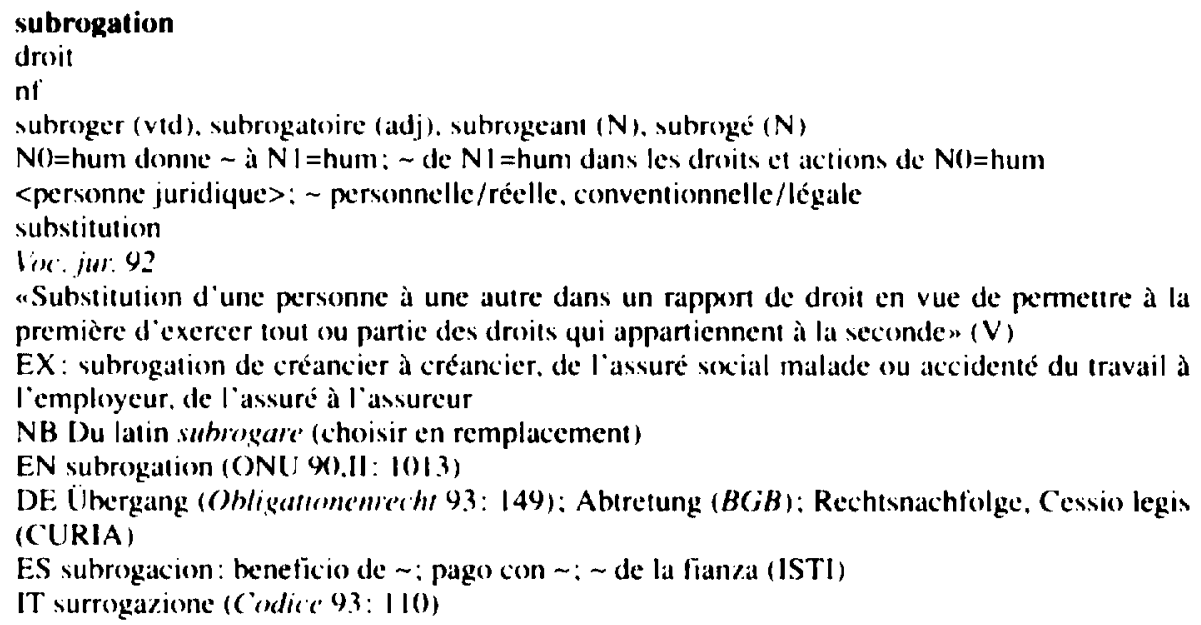

Enfin. la forme verbale se prête à un traitement tel que le suivant:

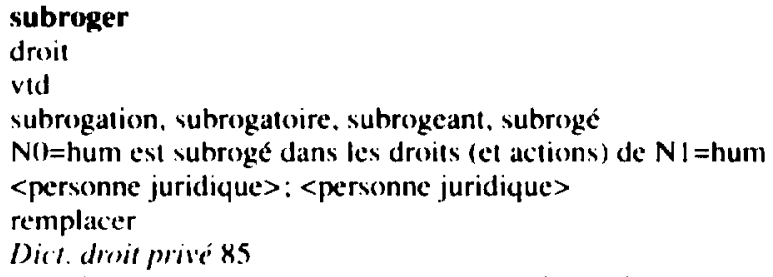


EN subrogate: "an insurer is subrogated to the rights of the insured on paying his claim" (Osborne 8.3): "The (ommunity shall acyuire any righos of action enjoyed by those concerned against third partiesm (CCF $8.3: 3(19)$

DF ubergehen: "der Sihadenersalt/an-pruch der Berechtigten gegen Dritte geht auf dic

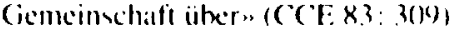

ES suhrogar

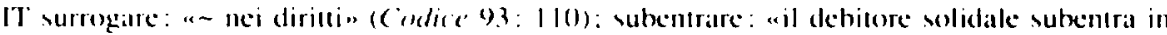

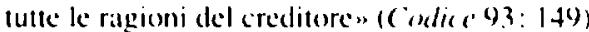

\section{CONCKPTIALISATION FI FXPERIENCE:}

Le vocabulaire des actes juridiques, administratifs el commerciaux est un vocabulaire de textes scoiaux. canoniques sinon stéréotypés, prévisibles sinon prérédigés. Les rédacteurs de documents à l'usage de partenaires structurels (assujettis, assurés, clients. contribuables etc.) ont avant tout la responsabilité de mettre en évidence les enjeux, les responsabilités, les garanties et less risques. On comprend que les organismes concernés privilégient le contact individuel pour personnaliser l'explication, et l'on voit aussi qu’à l'écrit on ne saurait faire l'ceconomic d'une définition et d"un ou plusieurs exemples.

Ce que montre également la terminographie multilingue, y compris la afausse multilingue", comme ici, est lat variabilité des conceptualisations dans les lextes corédigés dans plusieurs versions linguistiques officielles: en françaiss, la subrogation est présentée comme une affaire entre des persomeses a propos des droits de leune des deux. en anglais comme un accés à des droits d'unce autre personne. en allemand comme laceueil de ces droits par l'autre personne. Bien entendu il s'agit strictement de la même opération, mais vue et dite différemment: le scénario est le même. mais sa mise en scène langagière varie.

\section{CONCEPTIAI.ISATION FIT FORMLIIATION}

L'exemple considéré montre la relativité des aéquivalences», dont l'acceptabilité est évaluable seulement au niveau de l'énoncé global. Les formulations des mêmes normes juridiques se ressentent de différences en partic grammaticales et en partie culturelles qui donnent un contenu mi-scientifique mi-idéologique au concept de "génie de la langue".

Subrogation est un mot incommode qui conduit à une rédaction fortement figée ( " $x$ est subroge dans les droits de $y$ ", "la subrogation de $x$ dans les droits de $y$ », de façon tout à fait parallèle), voire à la redondance lexicale (puisque l'action en justice est un droit, l'expression les droits et actions ne s'impose pas en bonne logique).

L.anglais dispose du couple subrogate/subrogution au titre du même héritage latin (avec la mise au passif), mais il utilise concurremment une formulation active (ac quire).

L'allemand fait état du déplacement des droits eux-mémes, avec un verbe de mouvement dont le sujet est l'enjeu juridique, non les acteurs: autre façon d'être pragmatique.

L'italien. comme lespagnol et le français, recourt à la passivation du verbe et du nom d'action. mais il dispose aussi d'un verbe de mouvement à sujet humain yui dit merveilleusement les choses: subentrere. en conjoignant l'idée de substitution (sub) et celle d'accès, par une dérivation prélixale qui mime le contournement d'une difficulté tandis que la particule séparable de l'allemand signale un franchissement d'obstacle (ïhor).

\section{TERMINOCRAPHIE F.T TERMINONOMIE:}

La terminographic est menacéc par trois maux: la rigidité conceptuclle (un seul point de vue sur une technique vivante). la rigidité linguistique (un terme et un seul, avec dans le meilleur cas une collocation lexicale là où l'important est le mode d'emploi 
syntagmatique global, en langue spécialisée comme ailleurs), la rigidité bureaucratique (l'esprit de clocher exacerbé par la terminocratie). Chacun de ces aspects est développé dans Lerat (1995). mais pour ne pas sortir de notre propos, résumons ce que montre la façon de parler de la subrogation dans des documents grand public, dans des textes normatifs et dans des dictionnaires spécialisés.

La première leçon est le hesoin d'un effort d'explicitation maximale (linguistique et non linguistique). La consignation d'usages pluriels n'est pas seulement une exigence minimale en matière de scientificité mais aussi un gage de pluralisme et de tolérance. qui simpose tout particulièrement lorsque l'on a en vue, très légitimement, la promotion d'un usage plutôt que d'un autre. C'est également l'intérêt du traducteur, qui a besoin de savoir quelle clientèle préfère tel usage linguistique.

En matière d'expression, l'analyse ci-dessus montre que si un peu de terminologie éloigne de la linguistique, un travail terminologique plus approfondi y ramène, à la fois par la morphologie (ex: ir. subroger, fr. substirucr, it. subenucare, de. ubergehen) et par la syntaxe (ex: fr. "substitution d"une personne à une autre". en. "substitution of one person for another»).

Entin. pour ce qui est de la défense et illustration des langues, on se heurte ici à un réel que l'on ne saurait sans inconvénients perdre de vue durablement au profit de ce qui est plus voyant: la promotion de néologismes de substitution et de graphies nouvelles. Dans les sociétés développées, à plus forte raison dans les autres, autant l'importance du statut juridique des langues est vital. autant l'aménagement du corpus linguistique demande de la prudence. de l'attachement au patrimoine, un effort pédagogique massif en faveur de l'apprentissage conjoint des mots et des choses dans le corps social tout entier. Faute de quoi toute politique terminologique serait illusoire ou trompeuse.

\section{REFERENCWS}

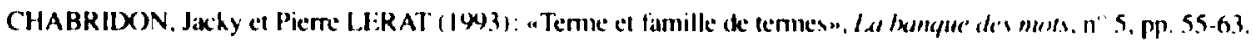

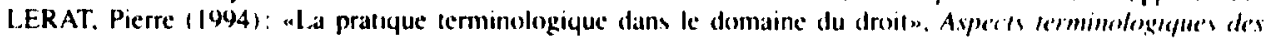

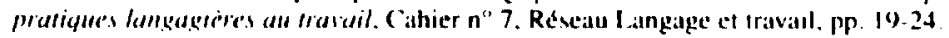

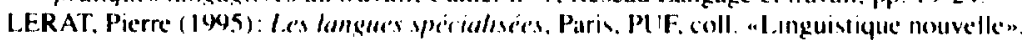

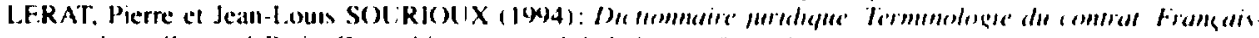

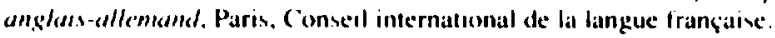

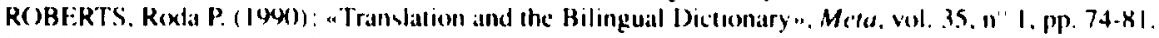

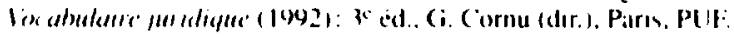

